\title{
Association of Blood Biomarkers of Bone Turnover in HIV-1 Infected Individuals Receiving Anti-Retroviral Therapy (ART)
}

\section{Najib Aziz ${ }^{1 *}$, Anthony W Butch ${ }^{2}$, Joshua J Quint ${ }^{1}$ and Roger Detels ${ }^{1,3}$}

${ }^{1}$ Department of Epidemiology, Fielding School of Public Health, University of California in Los Angeles, Los Angeles, CA, USA

${ }^{2}$ Department of Pathology \& Laboratory Medicine, University of California in Los Angeles, Los Angeles, CA, USA

${ }^{3}$ Department of Medicine, David Geffen School of Medicine, University of California in Los Angeles, Los Angeles, CA, USA

\begin{abstract}
Objective: To evaluate the association of bone turnover biomarkers with blood levels of alkaline phosphatase (ALP), bone-specific alkaline phosphatase (BAP), osteocalcin (OC), tartrate-resistant acid phosphatase (TRAP), parathyroid hormone (PTH), and other blood markers in HIV-1 infected men receiving anti-retroviral therapy (ART). Advances in the treatment of HIV-1 infection have extended the life span of HIV-1 infected individuals. However, these advances may come at the price of metabolic side effects and bone disorders, including premature osteopenia, osteoporosis and osteonecrosis.
\end{abstract}

Methods: Analyses of Ostase BAP, osteocalcin, and TRAP in blood were measured in three groups of MACS participants: 35 HIV-1 infected men on ART (A); 35 HIV-1- infected men not on ART (B); and 34 HIV-1 uninfected men (C).

Results: The mean and standard deviation results for groups $A, B$, and $C$ were $19.7 \pm 6.56,17.2 \pm 3.96$, and 16.9 \pm 5.78 for ostase BAP; $7.9 \pm 9.53,8.5 \pm 8.30$, and $5.5 \pm 1.65$ for osteocalcin; and $3.9 \pm 1.04,3.1 \pm 0.81$, and $2.5 \pm$ 0.59 for TRAP, respectively. Simple and multivariate analyses showed significant differences in mean TRAP and BAP concentrations between the three groups. In addition strong correlations between blood levels of Ostase BAP and TRAP ( $r=0.570, p=0.0004)$, and between blood levels of Ostase BAP and PTH $(r=0.436, P=0.0098)$ for HIV-1 infected men on ART were observed.

Conclusion: New strategies for measurement of blood and urine biochemical markers of bone formation and resorption during bone turnover can be useful for clinical monitoring of treatment of HIV-1 infected patients. Recently developed methods for measuring serum levels of TRAP and Ostase BAP represent superior laboratory tools for assessing the hyperactivity of osteoclasts, osteoblasts and bone loss in HIV-1 infected individuals receiving ART. Measurements of TRAP and BAP as bone turnover biomarkers are economical and are important for monitoring bone metabolism during ART and the need for osteoporosis treatment.

Keywords: Alkaline phosphatase; HIV infection; Osteocalcin; Osteoporosis; Parathyroid hormone; Tartrate-resistant acid phosphatase

\section{Introduction}

Bone cells are composed of four cell types: osteoblasts which form new bone, osteoclasts which control bone resorption, osteocytes, and bone lining cells. Bone turnover, or remodeling, is a dynamic process in which formation and resorption of bone maintain a dynamic equilibrium. In healthy, growing children, bone formation is favored over bone resorption. However, in healthy adults, bone remodeling is balanced until advanced age when bone resorption is slightly greater than formation. Age, metabolic conditions, diseases and medications can lead to an imbalance in the bone remodeling equilibrium [1].

Osteoporosis is "a systemic skeletal disease characterized by low bone mass and micro architectural deterioration of bone tissue with consequent increase in bone fragility and susceptibility to fracture" [2]. Excessive osteoclastogenesis and inadequate osteoblastogenesis are responsible for resorption of bone in postmenopausal and age-related (primary) osteoporosis [3]. The prevalence of osteoporosis in HIV-1 infected individuals (secondary osteoporosis) is more than three times higher than in HIV-1 uninfected individuals [4]. The use of long term ART in HIV-1 infected individuals has dramatically improved the quality of life and longevity of treated patients, but treatment is not without side effects and metabolic complications, including imbalances in bone metabolism [5].

Numerous pathophysiological disruptions in the body such as hormonal imbalances, inflammatory cytokines action, and kidney pathological processes may act on the skeleton and aggravate bone loss in HIV-1 infected persons. Osteoblast and osteoclast functions are influenced by a number of factors that are altered during HIV1 infection, including hormone levels, altered pro-inflammatory cytokines such as TNF- $\alpha$, expression of receptor activator NF- $\kappa B$ ligand (RANKL), osteoprotegerin (OPG), vitamin D, and calcium metabolism. The osteoclast is a member of the monocyte/macrophage family that is differentiated under the guidance of two critical cytokines, RANKL and M-CSF. An elevated RANKL/OPG ratio accelerates osteoclastogenesis and bone resorption [6].

Bone loss in HIV-1 infected individuals is one of several changes that can accelerate the normal aging process causing premature onset of cardiovascular, neurocognitive, bone degeneration diseases, and cancer [7]. ART is accompanied by increases in bone biomarker activation, which we hypothesize may offer insight into mechanisms underlying

*Corresponding author: Najib Aziz, Department of Epidemiology, Fielding Schoo of Public Health at UCLA 650 Charles E. Young Dr. South, Los Angeles, CA, USA 90095-1772; Fax: (310) 794-4092; E-mail: naziz@mednet.ucla.edu

Received July 02, 2014; Accepted September 29, 2014; Published October 10 2014

Citation: Aziz N, Butch AW, Quint JJ, Detels R (2014) Association of Blood Biomarkers of Bone Turnover in HIV-1 Infected Individuals Receiving Anti-Retrovira Therapy (ART). J AIDS Clin Res 5: 360. doi:10.4172/2155-6113.1000360

Copyright: (c) 2014 Aziz N, et al. This is an open-access article distributed unde the terms of the Creative Commons Attribution License, which permits unrestricted use, distribution, and reproduction in any medium, provided the original author and source are credited. 
bone loss in HIV-1 infected individuals. Studies have shown that bone loss in HIV-1 infected individuals occurs as a consequence of HIV viral protein, antiretroviral therapy, and vitamin D metabolism that has an effect on bone metabolism, especially on osteoclasts and osteoblasts, or indirectly through generalized inflammation promoting osteoblast apoptosis, reducing the functionality of these cells and promoting bone resorption [8-14].

In recent years, considerable advancement has been made in the measurement of bone biochemical markers that are considered to reflect specifically either bone formation or bone resorption. The gold standard for assessment of long term imbalances of bone loss or turnover in HIV-1 infected individuals or other conditions is densitometry techniques such as Dual energy $\mathbf{X}$-ray absorptiometry (DEXA) scans. On the other hand, the principal objective for using bone biomarkers is the detection of the imbalance itself and classification as low or high, decreased or increased bone turnover. Measurement of bone turnover blood biomarkers is non-invasive, inexpensive, and potentially useful when interpreted correctly [15]. Clinical findings and bone imaging techniques with blood biochemical tests can play an important role in the assessment, screening, diagnosis and follow-up of bone turnover caused by ART in HIV-1 infected individuals.

The aim of this study was to assess non-invasive and inexpensive routine laboratory bone blood biomarker tests that are not affected by age, feeding, collection time, or handling, and that could be used as a laboratory tool for monitoring bone formation and resorption changes which may be associated with osteoporosis in individuals diagnosed with HIV-1 infection and receiving ART. Therfore, we evaluated blood levels of bone turnover biomarkers in serum including total alkaline phosphatase (AP), Bone-specific AP (BAP), Osteocalcin (OC), and Tartrate-resistant acid phosphatase (TRAP), Parathyroid hormone (PTH), as well as CD4 and CD38 on CD8 levels as activation markers in HIV-1 infected men receiving and not receiving ART and uninfected men. We further examined correlation of those bone turnover biomarkers with other blood markers.

\section{Material and Methods}

\section{Specimens}

Overnight fasting blood samples were collected between 8:00 am and 11:00 am from most individual participating in the UCLA Multicenter AIDS Cohort Study (MACS) of the Natural History of AIDS in men who have sex with men [16] into $10 \mathrm{ml}$ plain tubes without anticoagulant (Becton Dickinson VACUTAINER Systems, Franklin Lakes, New Jersey, and USA) .

The institutional review board for human studies at UCLA approved the protocol (IRB\# 10-001677-CR-00003). Blood samples were obtained after informed consent. Serum was separated using standard technique and stored at $-70^{\circ} \mathrm{C}$ until analysis and serum samples were randomly selected from HIV-negative and positive men receiving and not receiving ART for the study.

\section{Ostase BAP assay}

Bone Alkaline phosphatase assay (Immunodiagnostics Systems Limited, NE35 9PD, and UK) is a solid phase, monoclonal antibody enzyme immunoassay that measures bone-specific alkaline phosphatase (BAP), and is an indicator of osteoblast activity in human serum. The assay was performed according to the manufacturer's instructions. Results were expressed in $\mu \mathrm{g} / \mathrm{mL}$, the lower limit of quantification was 1.5 $\mathrm{g} / \mathrm{L}$, inter-assay variability $(\mathrm{n}=10)$ for low and high samples was 7.8 and $6.5 \% \mathrm{CV}$ and intra-assay variability $(\mathrm{n}=4)$ were 4.7 and $9.9 \%$ respectively.

\section{Osteocalcin EIA}

The Metra osteocalcin assay (Quidel Corporation, San Diego, CA, USA) is a competitive immunoassay that measures intact osteocalcin in serum. The assay was performed according to the manufacturer's instructions. The results were expressed in $\mathrm{ng} / \mathrm{mL}$ and the lower limit of quantification was $1.1 \mathrm{ng} / \mathrm{ml}$, inter-assay variability $(\mathrm{n}=10)$ for low and high samples were 12 and $9 \% \mathrm{CV}$ and intra-assay variability $(\mathrm{n}=4)$ were 14.7 and $6.8 \% \mathrm{CV}$ respectively.

\section{Bone TRAP assay}

Tartrate-resistant acid phosphatase (TRAP) or TRACP 5b assay (Immunodiagnostics Systems Limited, NE35 9PD, UK) is a solid phase, monoclonal antibody enzyme immunoassay. It measures the active isoform $5 \mathrm{~b}$ of the tartrate-resistant acid phospahatase (TRAP) in serum and, is an indicator of bone resorption activity. All serum TRACP $5 b$ activity is derived from osteoclasts. Diurnal variability of serum TRACP $5 b$ activity is low and the levels are not affected by eating, allowing sample collection at any time of day. The assay was performed according to the manufacturer's instructions. Results were expressed in U/L. The lower limit of quantification was $0.25 \mathrm{U} / \mathrm{L}$, interassay variability $(\mathrm{n}=10)$ for low and high samples were 2.1 and $2.8 \% \mathrm{CV}$ and intra-assay variability $(\mathrm{n}=4)$ were 2.3 and $1.65 \% \mathrm{CV}$ respectively.

\section{Parathyroid hormone (PTH)}

Parathyroid hormone was measured using the Elecsys 2010 automated immunoanlyzer (Roche Diagnostics Corp, Indianapolis, IN, USA). The sandwich electrochemiluminescence assay was performed according to the manufacturer's instruction. Results were expressed in $\mathrm{pg} / \mathrm{mL}$ and the lower limit of quantification was $15.0 \mathrm{pg} / \mathrm{ml}$, inter-assay variability $(\mathrm{n}=10)$ for low and high samples were 2 and 3\% CV and intra-assay variability $(\mathrm{n}=10)$ were 10.7 and $12.6 \% \mathrm{CV}$ respectively.

\section{Blood ALP, AST, ALT, BUN, creatinine, inorganic Phosphorus and calcium}

The quantitative determination of Alkaline Phosphatase (ALP), Alanine Aminotransferase (ALT), Aspartate Aminotransferase (AST) inorganic Phosphorus $(\mathrm{P})$ and Calcium $(\mathrm{Ca})$ in human serum was preformed using a Beckman Coulter AU400 Chemistry analyzer according to the manufacturer instructions. During operation of the Beckman Coulter AU analyzer at least two levels of an appropriate quality control material were tested each day.

\section{Flowcytometry}

Flow cytometric immunophenotyping and analyses of CD38 on CD8 cells was performed using a FACSCalibur flow cytometer (Becton Dickinson, CA, U.S.A.). The CD38 relative fluorescence intensity (RFI) measurements were converted into the median number of molecules of CD38 per cell by multiplying by 34 , the approximate number of $\mathrm{PE}$ molecules detected per RFI channel on the flow cytometer [17].

\section{Statistical analysis}

A four-parameter curve-fitting program (Bio-Rad Laboratories, Irvine, CA) was used to generate calibration curves for the ELISA assays. SAS, version 9.3 (SAS Institute, Inc., Cary, North Carolina) was used for all descriptive and inferential statistics. Pearson and spearman correlation coefficients were calculated to assess the null hypothesis of no association between pairs of continuous variables in the overall study population. Analysis of variance was used to test the null hypothesis of no overall difference in means among all three study groups (F-test statistic) as well as pairwise comparison between 
study groups (t-test statistic) defined by ART and HIV status. When necessary, log transformations were used to induce normality and Welch's test was used to confirm that the estimates were not susceptible to unequal variances. Multivariate linear regression was used to adjust for potential confounders. Sigma Plot (Jandel Scientific, San Rafael, CA, USA) used for data plots.

\section{Results}

The majority of blood biomarkers had an approximately normal distribution with the exception of osteocalcin, ALT, AST, creatinine, and CD38. Results are presented in Table 1.

\section{Blood level of alkaline phosphatase (ALP)}

The median levels of alkaline phosphatase (ALP) were $64 \mathrm{U} / \mathrm{L}, 65$ $\mathrm{U} / \mathrm{L}$ and $73 \mathrm{U} / \mathrm{L}$ for HIV-1 uninfected men, HIV-1 infected men not on ART, and HIV-1 infected men on ART, respectively. There was not a statistically significant difference $(p=0.228)$ among the three groups.

\section{Blood level of Bone Alkaline phosphatase (BAP)}

The median levels of Ostase BAP were $15.8 \mu \mathrm{g} / \mathrm{L}, 16.4 \mu \mathrm{g} / \mathrm{L}$ and 20.9 $\mu \mathrm{g} / \mathrm{L}$ for HIV-1 uninfected men, HIV-1 infected men not on ART, and HIV-1 infected men on ART, respectively. Blood level of Ostase BAP was significantly higher for HIV-1 infected men on ART $(p=0.043)$ compared with HIV-1 uninfected men but was not significantly different in compared to HIV-1 infected men not on ART ( $\mathrm{p}=0.068)$ (Figure1a).

\section{Blood level of Osteocalcin (OC) or Bone gla protein}

The median levels of osteocalcin were $5.3 \mathrm{ng} / \mathrm{mL}, 6.9 \mathrm{ng} / \mathrm{mL}$, and $5.2 \mathrm{ng} / \mathrm{mL}$ for HIV-1 uninfected men, HIV-1 infected men not on ART,

\begin{tabular}{|c|c|c|c|c|c|c|}
\hline \multirow{2}{*}{ Variable } & \multirow{2}{*}{$\begin{array}{c}\text { Group A } \\
\qquad N=35\end{array}$} & \multirow{2}{*}{$\begin{array}{c}\text { Group B } \\
\qquad N=35\end{array}$} & \multirow{2}{*}{$\begin{array}{c}\text { Group C } \\
\qquad N=34\end{array}$} & \multicolumn{3}{|c|}{ P-value } \\
\hline & & & & $A=B$ & $A=C$ & $B=C$ \\
\hline AGE & $57(45-70)$ & $40(24-48)$ & $52(25-72)$ & $0.0001^{*}$ & 0.1429 & $0.0001^{*}$ \\
\hline HIV PCR copies /mL & $40(40-48000)$ & $42700(40-1850000)$ & N/A & $<0.001^{*}$ & N/A & N/A \\
\hline AST or SGOT (U/L) & $35.0(19-86)$ & $27.0(13-87)$ & $23.0(13-135)$ & $0.0200^{*}$ & $0.0064^{*}$ & 0.6184 \\
\hline ALT or SGPT (U/L) & $33.0(9-122)$ & $24.0(5-69)$ & $23(13-145)$ & $0.0104^{*}$ & $0.0310^{*}$ & 0.7368 \\
\hline BUN (mg/dL) & $16(9-33)$ & $14(9-38)$ & $15(7-29)$ & $0.0325^{*}$ & 0.1149 & 0.6155 \\
\hline Creatinine (mg/dL) & $1.0(0.7-1.7)$ & $1.0(0.6-2.1)$ & $1.0(0.7-1.3)$ & 0.4186 & 0.9932 & 0.4324 \\
\hline CD4 (cell/ $\mu \mathrm{L})$ & $533(73-1302)$ & $342(77-896)$ & $843(435-1319)$ & $0.0007^{*}$ & $0.0001^{*}$ & $0.0001^{*}$ \\
\hline CD38 (mol on CD8) & $1038(101-2191)$ & $734(321-2150)$ & $484(192-1019)$ & $0.0001^{*}$ & $0.0001^{*}$ & $0.0061^{*}$ \\
\hline ALP (U/L) & $73(25-130)$ & $65(50-145)$ & $64(33-108)$ & 0.2910 & 0.1060 & 0.2950 \\
\hline Ostase BAP ( $\mu \mathrm{g} / \mathrm{L})$ & $20.9(9.1-34.2)$ & $16.4(10.3-27.6)$ & $15.8(7.7-27.3)$ & 0.0678 & $0.0434^{*}$ & 0.8319 \\
\hline Osteocalin (ng/mL) & $5.2(1.0-42.5)$ & $6.9(3.8-54.3)$ & $5.3(3.1-9.4)$ & 0.7260 & 0.2070 & 0.1110 \\
\hline TRAP (U/L) & $3.9(2.5-6.7)$ & $2.9(1.2-4.7)$ & $2.5(1.6-4.7)$ & $0.0002^{*}$ & $0.0001^{*}$ & $0.0167^{*}$ \\
\hline PTH (pg/mL) & $24.7(8.3-47.7)$ & $23.0(12.5-145.2)$ & $31.6(16.4-65.9)$ & 0.1571 & $0.0313^{*}$ & 0.4245 \\
\hline Calcium (mg/dL) & $9.3(7.9-10.1)$ & $8.9(8.1-9.9)$ & $9.3(8.4-10.8)$ & 0.1136 & 0.6228 & 0.4090 \\
\hline Inorg. Phosphorus (mg/dL) & $3.0(1.7-3.8)$ & $3.3(2.5-6.0)$ & $3.0(2.1-4.2)$ & $0.0016^{*}$ & 0.1179 & 0.0978 \\
\hline
\end{tabular}

$\mathrm{A}=\mathrm{HIV}-1$ infected men on ART, B=HIV-1 infected men not ART, C=HIV-1uninfected men; N=number of men; mol=molecule; *=significant differences; and $\mathrm{N} / \mathrm{A}=$ not applicable;

Table 1: The median, minimum, maximum of blood markers under investigation for each group.
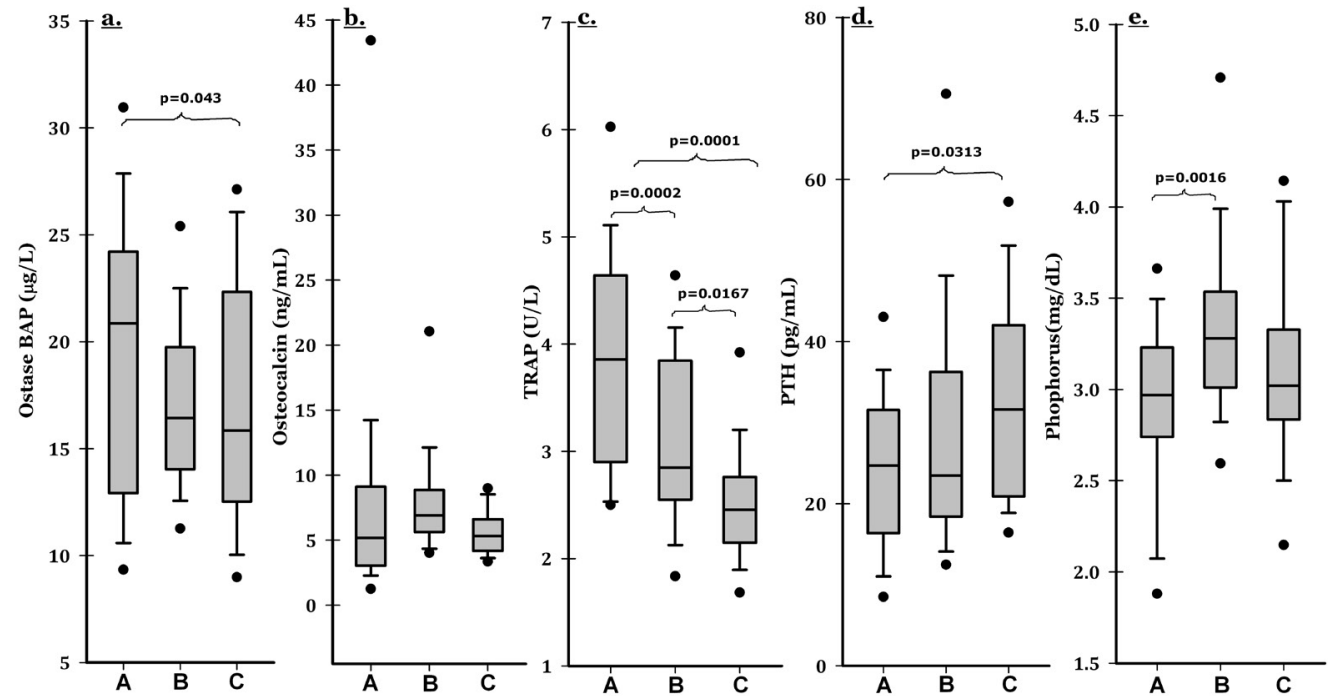

Figure 1: Levels of Ostase BAP (a), Osteocalcin (b), TRAP (c), PTH (d) and inorganic phosphorus(e) in HIV-1-infected men on ART (A), HIV-1-infected men not ART (B) and HIV-1-uninfected men (C). The solid lines represent the median, the box represents the $25^{\text {th }}$ to $75^{\text {th }}$ percentiles. The lower and upper horizontal bars represent the $5^{\text {th }}$ and $95^{\text {th }}$ percentiles, respectively. p-values represent the significant difference between group A and B; group B and C; and group A and C. 


\begin{tabular}{|l|c|c|c|c|}
\hline Model & $\begin{array}{c}\text { HIV-1-Infected men on } \\
\text { ART }\end{array}$ & $\begin{array}{c}\text { HIV-1-infected men* } \\
\text { Not on ART }\end{array}$ & p-value & HIV-1 Uninfected* men \\
\hline Unadjusted & 3.91 & -0.81 & $<0.0001$ & -1.38 \\
\hline Adjusted for Age & 4.06 & -0.85 & 0.0011 & -1.39 \\
\hline Adjusted for CD4/ $\mu \mathrm{L}$ & 3.98 & -0.81 & 0.0003 & -1.30 \\
\hline Adjusted for age \& CD4/ $\mu \mathrm{L}$ & 4.15 & -0.86 & 0.002 & $<0.0001$ \\
\hline
\end{tabular}

The first column represents the mean value for TRAP $(\mathrm{U} / \mathrm{L})$. ${ }^{*}$ The second and fourth columns represent the mean difference in TRAP comparing the HIV-1-infected to the control groups. Corresponding $\mathrm{p}$-values are provided for each estimate.

Table 2: Effect of antiretroviral therapy (ART) on TRAP, with adjustment for age and CD4/ $\mathrm{LL}$.

and HIV-1 infected men on ART respectively (Figure1b). Mean serum levels of osteocalcin were not significantly different between groups but after applying a $\log$ transformation, there was a significant difference between the three groups $(\mathrm{p}=0.0485)$.

\section{Blood level of tartrate-resistant acid phosphatase (TRAP or TRAcP)}

The median levels of TRAP were $2.5 \mathrm{U} / \mathrm{L}, 2.9 \mathrm{U} / \mathrm{L}$, and $3.9 \mathrm{U} / \mathrm{L}$ for HIV-1 uninfected men, HIV-1 infected men not on ART, and HIV-1 infected men on ART, respectively (Figure1c). The serum level of TRAP was significantly higher for HIV-1 infected men on ART compared with HIV-1-infected men not on ART $(\mathrm{p}=0.0002)$ and HIV-1-uninfected men $(\mathrm{p}=0.0001)$. The difference between groups was still evident after adjustment for age and CD4 count (Table 2).

\section{Blood level of parathyroid hormone (PTH)}

PTH is produced by chief cells of parathyroid glands and is an important regulator of calcium homeostasis. The median level of PTH were $31.6 \mathrm{pg} / \mathrm{ml} / \mathrm{L}, 23.0 \mathrm{pg} / \mathrm{l}$, and $24.7 \mathrm{pg} / \mathrm{mL}$ for HIV-1 uninfected men, HIV-1 infected men not on ART, and HIV-1 infected men on ART, respectively (Figure1d). The average serum level of PTH for HIV1 infected men on ART was 8.3 units lower than for HIV-1 uninfected men $(\mathrm{p}=0.031)$.

\section{Blood level of calcium and inorganic phosphorous}

The median levels of calcium were $9.3 \mathrm{mg} / \mathrm{dL}, 8.9 \mathrm{mg} / \mathrm{dL}$, and 9.3 $\mathrm{mg} / \mathrm{dL}$ for HIV-1 uninfected men, HIV-1 infected men not on ART, and HIV-1 infected men on ART, respectively. The serum level of calcium was within the reference range (8.7-10.5 $\mathrm{mg} / \mathrm{dL})$ and was not significantly different between groups ( $\mathrm{p}=0.1015)$.

The median levels of inorganic phosphorus were $3.02 \mathrm{mg} / \mathrm{dL}, 3.28$ $\mathrm{mg} / \mathrm{dL}$, and $2.97 \mathrm{mg} / \mathrm{dL}$ for HIV-1 uninfected men, HIV-1 infected men not on ART, and HIV-1 infected men on ART, respectively. The serum level of inorganic phosphorus was within the reference range (2.2-4.7 mg/dL). The serum level of phosphorus was significantly lower for HIV-1 infected men on ART compared with HIV-1 uninfected men $(\mathrm{p}=0.0016)$, and HIV-1 uninfected men $(\mathrm{p}=0.117)$ (Figure 1e).

\section{Relationship of biomarkers of bone turnover}

The correlation of blood levels of bone biomarkers with one another and with other blood markers were assessed to examine whether these markers are related, how strongly, and in what way. The results of the direction and strength of correlation in HIV-1 infected men on or not on ART and HIV-1 uninfected men are presented as follows:

HIV-1 infected on ART: Positive correlations were observed between blood levels of ALP and Ostase BAP ( $r=0.80, p<0.001)$, between ALP and TRAP $(r=0.47, p=0.005)$, between Ostase BAP and TRAP $(r=0.570, p=0.0004)$, between Ostase BAP and PTH $(r=0.44$, $\mathrm{p}=0.0098)$ between osteocalcin and inorganic phosphorus $(\mathrm{r}=0.38$, $\mathrm{p}=0.026)$, and between calcium and inorganic phosphorus $(\mathrm{r}=0.42$, $\mathrm{p}=0.011)$. A negative correlation was observed between blood levels of TRAP and calcium $(\mathrm{r}=-0.37, \mathrm{p}=0.298)$.

HIV-1 infected not on ART control group: Positive correlations were observed between blood levels of ALP and Ostase BAP ( $r=0.50$, $\mathrm{p}=0.003)$, between Ostase BAP and PTH $(\mathrm{r}=0.39, \mathrm{p}=0.022)$, between osteocalcin and TRAP $(\mathrm{r}=0.387, \mathrm{p}=0.022)$, and between calcium and inorganic phosphorus $(\mathrm{r}=0.64, \mathrm{p}<0.0001)$. No correlation was observed between blood levels of ALP and TRAP ( $r=0.17, \mathrm{p}=0.331$ ).

HIV-1 uninfected control group: Positive correlations were observed between blood levels of ALP and Ostase BAP ( $r=0.72$, $\mathrm{p}<0.0001)$, between ALP and TRAP $(\mathrm{r}=0.36, \mathrm{p}=0.041)$, between Ostase BAP and TRAP $(\mathrm{r}=0.574, \mathrm{p}=0.0004)$, and between Ostase BAP and PTH $(r=0.431, p=0.011)$. Also, there was negative correlation between blood levels of PTH and inorganic phosphorus $(r=-0.43, p=0.010)$.

\section{Discussion}

The present study explored the importance of bone biomarkers including blood levels of total alkaline phosphatase (ALP), bone specific alkaline phosphatase (BAP), and osteocalcin as markers of bone formation, blood tartrate-resistant acid phosphatase (TRAP) as a marker of bone resorption, and parathyroid hormone (PTH) as a calcium and phosphorus maintenance marker in HIV-1 infected men on and not on ART as well as uninfected men.

In a multivariate model, our data revealed significantly higher levels of TRAP ( $\mathrm{p}=0.0001$ ), a bone resorption marker, increases in the blood level of Ostase-BAP ( $p=0.0434)$, a bone formation marker, and significantly lower levels of PTH ( $\mathrm{p}=0.0313)$ among ART treated HIV-1 infected men. We also observed a positive correlation between bone turnover markers in HIV-1 infected men on ART with the strongest correlation between ALP and Ostase-BAP $(\mathrm{r}=0.80, \mathrm{p}=0.001)$, ALP and TRAP $(r=0.47, p=0.005)$, Ostase-BAP and TRAP $(r=0.57, p=0.0004)$, and between Ostase-BAP and PTH $(r=0.44, p=0.0098)$. There were no correlations observed between bone turnover biomarkers and other blood markers.

This data suggests that an imbalance observed in the rate of bone formation and bone resorption may cause the loss of bone mineral density (BMD) in HIV-1 infected men. All forms of acquired osteoporosis reflect increased osteoclast function relative to osteoblast production [18]. Increased levels of a blood bone resorption marker such as TRAP, indicates hyperactivity of osteoclasts which are responsible for bone resorption. Recent studies have suggested that TRAP, a widely used osteoclast marker, is localized in the transcytotic vesicles of resorbing osteoclasts and osteoclasts have developed efficient and unique machinery for dissolving mineral and degrading organic bone matrix rich in collagen fibers $[19,20]$.

Interestingly the blood level of TRAP in HIV-1 infected men on ART was 0.81 units higher than in the HIV-1 infected men not on 
ART $(\mathrm{p}<0.0001)$ and 1.38 units higher than in HIV-1 uninfected men $(\mathrm{p}<0.0001)$. Additionally, HIV-1 infected men not on ART had 0.57 units higher blood level of TRAP compared with HIV-1 uninfected men $(\mathrm{p}<0.0053)$. Adjusting for age and CD4 count did not change the relationships with TRAP. Therefore age and CD4 count do not appear to explain the elevated levels of TRAP.

Our data also shows no correlation between CD4 count and blood levels of bone biomarkers in HIV-1 infected men on ART. Conversely a published study indicated that low baseline absolute CD4 count is a strong and independent risk factor for bone loss after ART initiation [21].

The prevalence of low bone mineral density (BMD) is higher in HIV-1 infected individuals and worsens in men on ART [11]. Risk of osteoporosis is becoming relevant to daily clinical practice in HIV-1 infected aging persons especially for those receiving ART [22].

The significantly lower levels of total BMD in HIV-infected men on ART initiation is independent of the ART regimen or treatment strategy [23]. Fausto et al. observed no significant differences in biochemical markers of bone formation and resorption among HIV-1 infected individuals on ART and naïve patients, but found a positive correlation between high plasma level of HIV-RNA and osteoporosis [24]. In our study, there was no correlation between bone biomarkers and plasma HIV-RNA in the HIV-1 infected men not on ART and also there was no correlation between bone biomarkers and absolute CD4 number in all three groups. However, our data revealed significant differences in levels of blood biomarkers of bone formation (BAP) and resorption (TRAP) between HIV-1 infected men on ART and HIV-1 uninfected men (Table 1).

One study showed greater osteoclastic activity with bone loss in HIV1 infected patients on ART [25]. Teitelbaum suggests that hyperactivity of the osteoclasts relative to osteoblasts defines the pathophysiology of most cases of osteoporosis [26]. Our data also confirmed osteoblast and osteolclast hyperactivity in HIV-infected men on ART (Figure 1a-e). Fakruddin et al. suggested a mechanism by which HIV-1 or soluble HIV1 envelope gp120 can promote osteoclastogenesis and bone resorption via the induction of RANKL production by T-cells, as could be seen in active HIV replication with the shedding of gp160 [27]. We also noticed increased levels of ALP, Ostase-BAP, osteocalcin, and TRAP in HIV-1 infected men not on ART compared to HIV-1 uninfected men. Another study showed that the effect of azidothymidine (AZT) is mediated, at least in part, by enhancing RANKL-mediated osteoclastogensis and AZT-stimulated osteoclastogensis is mediated through activation of the TRAP promoter but requires RANKL [28]. Thus, the blood level of TRAP and BAP could be a suitable laboratory tool for evaluating osteoclast and osteoblast activities during ART treatment.

Ostase BAP is an isoform of ALP, which is involved in bone mineralization by cleaving organic phosphate and pyrophosphate (inhibitor of mineralization) and thereby, provides inorganic orthophosphate, a substrate for the synthesis of hydroxyapatite. It is a better marker of bone formation than ALP.

TRAP belongs to a family of acid phosphatases, of which at least five different isoforms are known. All acid phosphatases are inhibited by $\mathrm{L}(+)$ tartrate, except band 5 , which was therefore termed tartrate resistant acid phosphatase (TRAP or TRAcP). Band 5 has two subforms, $5 \mathrm{a}$ and $5 \mathrm{~b}$. TRAP-5b is characteristic of osteoclasts. It is an iron containing lysosome enzyme synthesized by osteoclasts and is localized in the transcytotic vesicles of resorbing osteoclasts. It can generate highly destructive reactive oxygen species (ROS) that are able to destroy collagen. The ROS generating activity of TRAP may have an important role in both bone resorption and in the immune defense system [20].

Biochemical markers of bone turnover change much faster than BMD in HIV-1 infected individuals and also change rapidly in response to therapeutic interventions in osteoporosis patients.

Changes in BMD in the HIV-infected individuals on ART occur slowly and are usually not detectable for several years, at which time a single measurement of a biochemical marker cannot predict the absolute rate of bone loss in a patient. Therefore, measurement of bone turnover markers, especially blood levels TRAP and BAP, are an excellent laboratory tool for evaluating rapid bone loss in HIV-1 infected and treated patients.

DEXA is the most commonly used gold standard screen test for measuring BMD. It uses two very low dose $\mathrm{x}$-ray beams with different energy levels. The HIV-1 infected on ART related BMD changes occur very slowly and may not reliably be detected early by DEXA. In contrast, changes in blood bone turnover markers can be observed sooner and are easily measured. Measuring and re-measuring those markers without radiation is an excellent, non-invasive laboratory tool for clinicians monitoring follow-up of their patients. Further the predictive value of DEXA for BMD evaluation can be enhanced when used in combination with biochemical indices of bone resorption.

To our knowledge this will be the first data to be published about blood levels of TRAP as a bone resorption marker and its association with HIV-1 infected men on and not on ART and HIV-1 uninfected men. The data may offer insights into mechanisms underlying bone loss in those patients and may reveal that in HIV-1 infected men, bone resorption is occurring faster than bone formation during ART.

\section{Conclusion}

The hyperactivity of osteoclastogensis signaled by increased levels of TRAP could be the principal cause of bone resorption and low BMD in HIV-1 infected men. The blood level of TRAP can therefore be an important marker for predicting bone matrix degradation in patients. Serum level of TRAP and BAP provide useful information about bone metabolism activity during ART. Treatment of HIV-1 infected individuals with ART appears to further increase the higher rate of bone turnover observed in HIV-infected patients. Frequent measurement of the blood levels of these biomarkers can be used by clinicians for monitoring the possible onset of osteoporosis in HIV1 infected patients undergoing ART treatment. Further longitudinal studies of HIV-1 infected men receiving ART are needed to confirm the use of these blood markers in predicting osteoporosis.

\section{Acknowledgements}

We thank the men who participate in the MACS, who make this and many other studies possible. Also we thank John Oishi for recruitment coordination; Kevin Barrett for data; and Shaun Hsueh, Yegermal Asnake and Timothy Ryner for laboratory assistance and manuscript preparation. Data in this manuscript was collected by the Multicenter AIDS Cohort Study with center at University of California, Los Angeles (PIs: Roger Detels, Oto Martinez-Meza). This work was supported by grants from the National Institutes of Health (U01-A1-35042), The MACS is funded by the National Institute of Allergy and Infectious Diseases, with additional supplemental funding from the National Cancer Institute (U01-AI35042, 5-M01-RR00722 (GCRC), U01-A135043, U01-Al37984, U01-A135039, U01Al35040, U01-AI37613, U01-Al35041). This project has been funded in whole or in part with Federal funds from the National Cancer Institute, National Institutes of Health, under Contract No. N01-CO56000 
Citation: Aziz N, Butch AW, Quint JJ, Detels R (2014) Association of Blood Biomarkers of Bone Turnover in HIV-1 Infected Individuals Receiving AntiRetroviral Therapy (ART). J AIDS Clin Res 5: 360. doi:10.4172/2155-6113.1000360

\section{References}

1. Marks SC Jr, Odgren PR (2002) Structure and Development of the Skeleton In Principles of Bone Biology ( $2^{\text {nd }}$ Edn., volume1), Academic Press, San Diego, San Francisco, New York, Boston, London, Sydney, Tokyo.

2. Kanis JA (2002) Diagnosis of osteoporosis and assessment of fracture risk Lancet 359: 1929-1936.

3. Manolagas SC, Jilka RL (1995) Bone marrow, cytokines, and bone remodeling Emerging insights into the pathophysiology of osteoporosis. New England Journal Medicine. 332: 305-311

4. Brown TT, Qaqish RB (2006) Antiretroviral therapy and the prevalence of osteopenia and osteoporosis: a meta-analytic review. AIDS 20: 2165-2174.

5. Ofotokun I, McIntosh E, Weitzmann MN (2012) HIV: inflammation and bone. Curr HIVIAIDS Rep 9: 16-25.

6. Vikulina T, Fan X, Yamaguchi M, Roser-Page S, Zayzafoon M, et al. (2010) Alterations in the immuno-skeletal interface drive bone destruction in HIV-1 transgenic rats. Proc Natl Acad Sci U S A 107: 13848-13853.

7. Deeks SG (2009) Immune dysfunction, inflammation, and accelerated aging in patients on antiretroviral therapy. Top HIV Med 17: 118-123.

8. Amorosa V, Tebas P (2006) Bone disease and HIV infection. Clin Infect Disease. 42:108-114

9. Haskelberg H, Carr A, Emery S (2011) Bone turnover markers in HIV disease. AIDS Rev 13: 240-250

10. Mondy K, Yarasheski K, Powderly WG, Whyte M, Claxton S, et al. (2003) Longitudinal evolution of bone mineral density and bone markers in human immunodeficiency virus-infected individuals. Clin Infect Dis 36: 482-490.

11. Ofotokun I, Weitzmann MN (2010) HIV-1 infection and antiretroviral therapies: risk factors for osteoporosis and bone fracture. Curr Opin Endocrinol Diabetes Obes 17: 523-529.

12. Powderly WG (2012) Osteoporosis and bone health in HIV. Curr HIVIAIDS Rep 9: 218-222.

13. Rothman MS, Bessesen MT (2012) HIV infection and osteoporosis: pathophysiology, diagnosis, and treatment options. Curr Osteoporos Rep 10: 270-277.

14. Saccomanno MF, Ammassari A (2011) Bone disease in HIV infection. Clin Cases Miner Bone Metab 8: 33-36.
15. Seibel MJ (2006) Biochemical markers of bone turnover part II: clinical applications in the management of osteoporosis. Clin Biochem Rev 27: 123138.

16. Detels R, Jacobson L, Margolick J, Martinez-Maza O, Muñoz A, et al. (2012) The multicenter AIDS Cohort Study, 1983 to â€!. Public Health 126: 196-198.

17. Hultin LE, Matud JL, Giorgi JV (1998) Quantitation of CD38 activation antigen expression on CD8+ T cells in HIV-1 infection using CD4 expression on CD4+ T lymphocytes as a biological calibrator. Cytometry 33: 123-132.

18. Teitelbaum SL (2007) Osteoclasts: what do they do and how do they do it? Am J Pathol 170: 427-435.

19. Halleen JM, Räisänen SR, Alatalo SL, Väänänen HK (2003) Potential function for the ROS-generating activity of TRACP. J Bone Miner Res 18: 1908-1911.

20. Väänänen HK, Zhao H, Mulari M, Halleen JM (2000) The cell biology of osteoclast function. J Cell Sci $113: 377-381$

21. Grant PM, Kitch D, McComsey GA, Dube MP, Haubrich R, et al. (2013) Low baseline CD4+ count is associated with greater bone mineral density loss after antiretroviral therapy initiation. Clin Infect Dis 57: 1483-1488.

22. Tebas P, Powderly WG, Claxton S, Marin D, Tantisiriwat W, et al. (2000) Accelerated bone mineral loss in HIV-infected patients receiving potent antiretroviral therapy. AIDS 14: F63-67.

23. Brown TT, Chen Y, Currier JS, Ribaudo HJ, Rothenberg J, et al. (2013) Body composition, soluble markers of inflammation, and bone mineral density in antiretroviral therapy-naive HIV-1-infected individuals. J Acquir Immune Defic Syndr 63: 323-330.

24. Fausto A, Bongiovanni M, Cicconi P, Menicagli L, Ligabò EV, et al. (2006) Potential predictive factors of osteoporosis in HIV-positive subjects. Bone 38 : 893-897.

25. de Menezes Barbosa EG, de Paula FJ, Machado AA, de Assis Pereira $F$, Barbosa Júnior $F$, et al. (2013) Impact of antiretroviral therapy on bone metabolism markers in HIV-seropositive patients. Bone 57: 62-67.

26. Teitelbaum SL (2000) Bone resorption by osteoclasts. Science 289: 1504-1508.

27. Fakruddin JM, Laurence J (2003) HIV envelope gp120-mediated regulation of osteoclastogenesis via receptor activator of nuclear factor kappa B ligand (RANKL) secretion and its modulation by certain HIV protease inhibitors through interferon gamma/RANKL cross-talk. J Biol Chem 278: 48251-48258.

28. Pan G, Wu X, McKenna MA, Feng X, Nagy TR, et al. (2004) AZT enhances osteoclastogenesis and bone loss. AIDS Res Hum Retroviruses 20: 608-620. 